\title{
La multiplication de Rajchman et les ensembles $U(\varepsilon)$ de Zygmund
}

\author{
by \\ JeAn-Pierre Kahane (Orsay)
}

Sommaire. Le but de cet article est de donner une construction simple d'ensembles $U(\varepsilon)$ de Zygmund dont le complémentaire est de mesure nulle. La multiplication formelle des distributions, issue de Rajchman, est l'outil essentiel. L'histoire du sujet est esquissée à la fin de l'article.

1. La multiplication de Rajchman. Pour des séries formelles

$$
S=\sum_{n=-\infty}^{\infty} a_{n} z^{n}
$$

à coefficients positifs $\left(a_{n} \geq 0\right)$, la multiplication correspond à la convolution des suites de coefficients :

$$
S S^{\prime} S^{\prime \prime}=\sum_{n=-\infty}^{\infty}\left(\sum_{m+m^{\prime}+m^{\prime \prime}=n} a_{m} a_{m^{\prime}} a_{m^{\prime \prime}}\right) z^{n} .
$$

Nous dirons qu'elle est bien définie si les coefficients du produit sont finis.

À une série formelle à coefficients quelconques $\left(a_{n} \in \mathbb{C}\right)$ nous associons son "comodule"

$$
"|S| "=\sum_{n=-\infty}^{\infty}\left|a_{n}\right| z^{n} .
$$

Nous dirons qu'un produit de séries formelles est bien défini si le produit de leurs comodules est bien défini. De cette façon, la multiplication des séries formelles est associative et commutative.

On pose maintenant :

$$
z^{n}=e^{2 \pi i n t}=e_{n}(t) \quad(n \in \mathbb{Z}, t \in \mathbb{T})
$$

et on s'intéresse aux séries $S$ qui représentent des distributions sur $\mathbb{T}$. On identifie $S$ et la distribution qu'elle représente, et on traduit la multiplica-

2000 Mathematics Subject Classification: 42A63, 43A46. 
tion formelle des séries en multiplication formelle des distributions : c'est la multiplication de Rajchman.

Dans cet article les seules distributions qui interviendront sont des pseudomesures, c'est-à-dire des distributions à coefficients bornés :

$$
S=\sum a_{n} e_{n}, \quad \sup \left|a_{n}\right|<\infty,
$$

et nous écrirons également

$$
S(t)=\sum a_{n} e_{n}(t)
$$

pour mettre en évidence la variable, sans que cette notation ait nécessairement un sens pour une valeur donnée de $t$. On dit que $S$ est une pseudofonction si les coefficients tendent vers zéro et on désigne par PF l'espace des pseudofonctions, normé par

$$
\|S\|_{\mathrm{PF}}=\sup \left|a_{n}\right| \quad\left(\left(a_{n}\right) \in c_{0}(\mathbb{Z})\right) .
$$

On peut multiplier des pseudofonctions et des fonctions de la classe $A$ de Wiener (correspondant à $\left(a_{n}\right) \in l^{1}(\mathbb{Z})$ ). Le produit est une pseudofonction, et

$$
\|S f\|_{\mathrm{PF}} \leq\|S\|_{\mathrm{PF}}\|f\|_{A} .
$$

Nous nous proposons d'associer à toute pseudofonction $S$ une mesure de probabilité $\mu$, portée par un ensemble fermé $E$ de mesure de Lebesgue nulle, et telle que le produit $S \mu$ soit bien défini et soit une pseudofonction. Voici les étapes. Suivant l'usage, les coefficients d'une pseudomesure $S$ sont notés $\widehat{S}(n)$ (donc, ci-dessus, $a_{n}=\widehat{S}(n)$ ). Les coefficients de " $|S|$ " sont les $|\widehat{S}(n)|$.

LEMme 1. Soit $V \in \mathrm{PF}$ et $h \in A$, toutes deux de type positif $(\widehat{V}(n) \geq 0$ et $\widehat{h}(n) \geq 0$ pour tout $n$ ), avec $\widehat{h}(0)=0$. On donne $\alpha>0$ (petit) et $a>0$ (grand). Il existe $l_{0}=l_{0}(V, h, \alpha, a)$ tel que, pour $l \geq l_{0}$ entier, le produit $V(t) h(l t)$ soit une pseudofonction telle que

$$
\begin{gathered}
\|V(t) h(l t)\|_{\mathrm{PF}} \leq\|V\|_{\mathrm{PF}}\|h\|_{\mathrm{PF}}+\alpha, \\
\left(V(t) h(l t)^{\wedge}(n) \leq \alpha \quad \text { lorsque }-a \leq n \leq a .\right.
\end{gathered}
$$

La preuve est immédiate en regardant les coefficients. Dans la première inégalité, l'hypothèse $\widehat{h}(0)=0$ ne joue aucun rôle, et le second membre fait intervenir la norme de $h$ comme pseudofonction. L'hypothèse $\widehat{h}(0)=0$ est essentielle dans la seconde inégalité.

Lemme 2. On donne $T \in \mathrm{PF}$, de type positif, et une suite $\left(h_{j}\right)$ dans $A$ $(j=1,2, \ldots)$, toutes de type positif, et de valeur moyenne nulle $\left(\widehat{h}_{j}(0)=\right.$ $0)$, telles que les normes $\left\|h_{j}\right\|_{\mathrm{PF}}$ tendent vers 0 . Il existe des fonctions $l_{j}\left(x_{1}, \ldots, x_{j-1}\right)(j=1,2, \ldots)$ telles que, pour $l_{j} \geq l_{j}\left(l_{1}, \ldots, l_{j-1}\right)(j=$ $1,2, \ldots)$, le produit 


$$
T(t) \prod_{j=1}^{\infty}\left(1+h_{j}\left(l_{j} t\right)\right)
$$

soit bien défini et soit une pseudofonction.

La preuve s'effectue par récurrence en utilisant le lemme 1 avec

$$
V(t)=T(t) \prod_{j=1}^{n-1}\left(1+h_{j}\left(l_{j} t\right)\right), \quad h(t)=h_{n}(t) .
$$

Le détail est laissé au lecteur.

LEMME 3. On donne $T \in \mathrm{PF}$, de type positif, et une suite $\left(g_{j}\right)$ dans $A$ $(j=1,2, \ldots)$, telles que $\widehat{g}_{j}(0)=0, g_{j}(t) \geq-1$ pour tout $t, g_{j}(t)=-1$ sur un ensemble $E_{j}$ dont la mesure de Lebesgue est $1 /(j+1)(j=1,2, \ldots)$, et $\left\|g_{j}\right\|_{\mathrm{PF}}=O(1 / j)(j \rightarrow \infty)$. Il existe alors des entiers $l_{j}$ tels que le produit

$$
\prod_{j=1}^{\infty}\left(1+g_{j}\left(l_{j} t\right)\right)
$$

définisse une mesure $\mu$ dont le support a une mesure de Lebesgue nulle, et telle que le produit de son comodule par $T$ soit une pseudofonction:

$$
T^{*}|\mu| " \in \mathrm{PF} \text {. }
$$

Preuve. Pour que le produit infini définisse une mesure positive portée par un fermé de mesure de Lebesgue nulle, il suffit que les rapports $l_{j+1} / l_{j}$ soient assez grands. Pour que $T$ " $|\mu|$ " soit bien défini et soit une pseudofonction il suffit de pouvoir appliquer le lemme 2 avec $h_{j}={ }^{~ "}\left|g_{j}\right|$ ", il suffit donc que $l_{j}$ soit assez grand quand $l_{1}, \ldots, l_{j-1}$ sont donnés. Ces deux conditions sont compatibles, ce qui démontre le lemme 3.

ThÉORÈme 1. On donne $T \in \mathrm{PF}$, de type positif. Il existe une mesure de probabilité $\mu$, portée par un fermé de mesure de Lebesgue nulle, telle que le produit T" $T|\mu| "$ soit bien défini et soit une pseudofonction. Si $S \in \mathrm{PF}$ et $|\widehat{S}(n)| \leq \widehat{T}(n)$ pour tout $n, S \mu$ est une pseudofonction portée par le support de $\mu$.

Preuve. Le lemme 3 donne la première partie du théorème. Pour la seconde, il suffit de montrer que $S \mu \varphi=0$ lorsque $\varphi \in A$ et que les supports de $\varphi$ et de $\mu$ sont disjoints. Il en est bien ainsi parce qu'alors $S \varphi$ est une pseudofonction portée par le support de $\varphi$, donc ([3], p. 54, proposition 1) les sommes partielles symétriques de sa série de Fourier convergent uniformément vers 0 sur le support de $\mu$, donc $S \varphi \mu=0$.

2. Les ensembles d'unicité $U(\varepsilon)$ de Zygmund. Dans la théorie de l'unicité du développement trigonométrique, on appelle ensemble d'unicité, 
ou ensemble $U$, tout sous-ensemble $E$ de $\mathbb{T}$ tel que la seule série trigonométrique convergeant vers 0 hors de $E$ (au sens des sommes partielles symétriques) soit la série nulle. On démontre aisément que la mesure de Lebesgue d'un ensemble $U$ est nulle.

Étant donné une suite $\left(\varepsilon_{n}\right)$ positive $(n \in \mathbb{Z})$ telle que

$$
\varepsilon_{n}=o(1) \quad(|n| \rightarrow \infty)
$$

on appelle ensemble $U(\varepsilon)$, suivant Zygmund, tout sous-ensemble $E$ de $\mathbb{T}$ telle que la seule série trigonométrique $\sum a_{n} e_{n}(t)$ dont les coefficients vérifient $a_{n}=O\left(\varepsilon_{n}\right)(n \rightarrow \infty)$ et dont les sommes partielles symétriques tendent vers 0 quand $t \notin E$ soit la série nulle. Nous allons montrer, en simplifiant l'argument de [2], qu'il existe des ensembles $U(\varepsilon)$ de mesure pleine.

Posons $T=\sum \varepsilon_{n} e_{n}$ et $S=\sum a_{n} e_{n}$. L'hypothèse est

$$
\widehat{S}(n)=O(\widehat{T}(n)) \quad(|n| \rightarrow \infty) .
$$

Désignons par $S_{m}(m=1,2, \ldots)$ les sommes partielles symétriques

$$
S_{m}(t)=\sum_{|n| \leq m} \widehat{S}(n) e_{n}(t) .
$$

Soit $D$ un ensemble dénombrable dense sur $\mathbb{T}$, et soit $E_{0}$ le support de la mesure $\mu$ du théorème 1 . Soit enfin $E=E_{0}+D$ (somme algébrique sur $\mathbb{T}$ ). Observons que la mesure de Lebesgue de $E$ est nulle.

ThÉorème 2. Si $S_{m}(t)$ tend vers $0(m \rightarrow \infty)$ quand $t \in E$, S est la série nulle.

Preuve. Utilisons d'abord le fait que $S_{m}(t)$ tend vers 0 quand $t \in E_{0}$. Soit $F$ le support de $S \mu$, qui est contenu dans $E_{0}$ d'après le théorème 1 . Supposons d'abord $F \neq \emptyset$. D'après le théorème de Baire, il existe une portion $F^{\prime}$ de $F$ (c'est-à-dire une intersection non vide de $F$ et d'un intervalle ouvert $I$ ) sur laquelle les $S_{m}(t)$ sont uniformément bornées. D'après le théorème de Lebesgue,

$$
\lim _{m \rightarrow \infty} \int_{F^{\prime}} S_{m}(t) d \mu(t)=0 .
$$

De même, pour toute fonction $f$ de la classe $A$ à support dans $I$,

$$
\lim _{m \rightarrow \infty} \int f(t) S_{m}(t) d \mu(t)=0 .
$$

D'après le théorème $1, S_{m} \mu$ tend vers $S \mu$ dans $\mathrm{PF}$. Il en résulte que $(f S \mu)^{\wedge}(0)=0$ pour toute $f \in \mathcal{A}$ portée par $I$, donc $S \mu=0$ sur $I$, contrairement à la définition de $F^{\prime}$. Donc $F=\emptyset$, c'est-à-dire $S \mu=0$, et en particulier

$$
\sum \widehat{S}(n) \widehat{\mu}(-n)=(S \mu)^{\wedge}(0)=0
$$


Utilisons maintenant le fait que $S_{m}(t+s)$ ) tend vers 0 quand $t \in E_{0}$ et $s \in D$. Il en résulte

$$
\sum \widehat{S}(n) \widehat{\mu}(-n) e_{n}(s)=0 \quad(s \in D) .
$$

La série est absolument convergente d'après le théorème 1 . Comme $D$ est dense, elle est nulle, donc $\widehat{S}(0)=0$. En remplaçant $S$ par $S e_{k}(k \in \mathbb{Z})$, on a $\widehat{S}(-k)=0$, donc $S$ est la série nulle.

L'ensemble $\mathbb{T} \backslash E$ est bien un $U(\varepsilon)$ de mesure pleine.

3. Esquisse de l'histoire du sujet. La théorie de l'unicité du développement trigonométrique, contenue en germe dans la thèse de Riemann (1854), remonte à Cantor (1870). Cantor a montré que les ensembles réductibles (dénombrables, à fermeture compacte) sont des ensembles $U$ (1872), Young que le résultat vaut pour tous les dénombrables (1909), Nina Bari et Zygmund, indépendamment, que toute réunion dénombrable d'ensembles $U$ fermés est un ensemble $U$ (1923). Comme nous l'avons dit, tout ensemble $U$ est de mesure de Lebesgue nulle. Mais la réciproque est inexacte (Menchoff, 1916). Il existe des ensembles $U$ non dénombrables, par exemple l'ensemble triadique de Cantor (Rajchman, 1922).

Les outils essentiels, pour étudier les séries trigonométriques à coefficients tendant vers 0 , sont l'intégration formelle et la multiplication formelle par des fonctions assez régulières. C'est la "théorie de Riemann", développée par Rajchman et par Zygmund, et exposée au chapitre 9 du livre de Zygmund [5]. C'est Rajchman qui a systématisé l'usage de la multiplication formelle. La traduction en termes de pseudomesures et de pseudofonctions se trouve dans le chapitre 5 de [3].

Zygmund a introduit les ensembles $U(\varepsilon)$ en 1926 [4]. Il a démontré l'existence d'ensembles $U(\varepsilon)$ fermés dont la mesure de Lebesgue est aussi proche qu'on le veut de celle du cercle, et posé deux questions : 1) existe-t-il des ensembles $U(\varepsilon)$ de mesure pleine ? 2) la réunion dénombrable d'ensembles $U(\varepsilon)$ fermés est-elle nécessairement un ensemble $U(\varepsilon)$ ? Dans ces deux questions, $\varepsilon$ est une suite $\left(\varepsilon_{n}\right)$ donnée, tendant vers 0 quand $|n| \rightarrow \infty$. Une réponse positive à la seconde question, compte tenu du théorème établi par Zygmund, entraînerait une réponse positive à la première ; mais elle est encore en question.

L'existence d'ensembles $U(\varepsilon)$ de mesure pleine a été établie par Kahane et Katznelson en 1973 [2], et des précisions sur les mesures et dimensions de Hausdorff de leurs complémentaires ont été fournies par Bernard Connes en 1976 [1]. Depuis lors, je n'ai pas connaissance de progrès sur ce sujet.

Il est courant, dans ces questions, d'imposer à la suite $\left(\varepsilon_{n}\right)$ des conditions supplémentaires : parité, décroissance à droite (pour $n>0$ ), convexité à droite, régularité de diverses sortes. Ce n'était pas nécessaire ici. 
La note [2] utilise une mesure $\mu$, portée par un fermé de mesure de Lebesgue nulle, et telle que

$$
\sum|\widehat{\mu}(-n)| \varepsilon_{n}<\infty
$$

Elle pose une question : si l'on suppose que la série trigonométrique $\sum a_{n} e_{n}(t)$, où $a_{n}=O\left(\varepsilon_{n}\right)$, converge vers 0 (au sens des sommes partielles symétriques) lorsque $t$ appartient au support de $\mu$, s'ensuit-il que

$$
\sum \widehat{\mu}(-n) a_{n}=0 \quad ?
$$

C'est en examinant cette question de nouveau que, pour aboutir aisément à la conclusion, j'ai modifié la condition imposée à $\mu$, sous la forme

$$
\lim _{|k| \rightarrow \infty} \sum|\widehat{\mu}(-n)| \varepsilon_{n+k}=0,
$$

chacune des sommes écrites étant finie. La traduction est $T^{\text {" }}|\mu| " \in \mathrm{PF}$, et l'existence de cette mesure $\mu$ est l'objet du théorème 1 . Le passage du théorème 1 au théorème 2 est directement inspiré de [2].

\section{Références}

[1] B. Connes, Mesure de Hausdorff des complémentaires des $U_{\varepsilon}$ de Zygmund, Israel J. Math. 23 (1976), 1-7.

[2] J.-P. Kahane et Y. Katznelson, Sur les ensembles d'unicité $U(\varepsilon)$ de Zygmund, C. R. Acad. Sci. Paris Sér. A 277 (1973), 893-895.

[3] J.-P. Kahane et R. Salem, Ensembles parfaits et séries trigonométriques, Hermann, Paris 1963 ; 2ème édition, 1994.

[4] A. Zygmund, Contribution à l'unicité du développement trigonométrique, Math. Z. 24 (1926), 40-46. (Selected Papers I (1989), 16-22.)

[5] - - Trigonometric Series, Vol. I, Cambridge Univ. Press, 1959.

Département de Mathématiques

Université Paris-Sud

Bâtiment 425

91405 Orsay Cedex, France

E-mail: Jean-Pierre.Kahane@math.u-psud.fr 